\title{
Energy Efficiency Management and Route Optimization for Wireless Sensor Network under the Ubiquitous Power Internet of Things
}

\author{
Yi Zhang $^{1,2^{*}}$ \\ ${ }^{1}$ School of Economics and Management, Tongji University, Shanghai 200090, China \\ ${ }^{2}$ School of Economics and Management, Shanghai University of Electric Power, Shanghai 200090, China
}

Corresponding Author Email: zhangyish@126.com

https://doi.org/10.18280/ejee.210213

Received: 20 January 2019

Accepted: 2 April 2019

\section{Keywords:}

ubiquitous power internet of things (UPIoT), energy consumption model, node quality, route optimization

\begin{abstract}
The traditional routing algorithms for data transmission in the wireless sensor network (WSN) under the ubiquitous power Internet of Things (UPIoT) are faced with two common defects, namely, the imbalance between the nodes in energy consumption and the premature death of many nodes. To solve the problems, this paper puts forward a method to manage the energy efficiency and optimize the route for the WSN under the UPIoT. Firstly, an energy consumption model of network nodes was established after analyzing the causes of node energy loss, which effectively reduces the energy consumed by each node during the clustering process. Next, the author designed a way to compute node quality, and selected cluster heads by node quality, such that the energy consumption is balanced across the WSN. On this basis, a node clustering routing algorithm was presented to dynamically adjust the communication radii of cluster heads and general nodes, thus extending the lifecycle of the WSN under the UPIoT. The simulation results show that our method outperformed the traditional clustering routing algorithm in controlling energy loss of nodes and enhancing network reliability and stability. The research findings provide a valuable reference for the efficient data transmission of the WSN under the UPIoT.
\end{abstract}

\section{INTRODUCTION}

As the top legislature and political advisory body of China convened in 2019, the State Grid Corporation of China (SGCC), committed to become a world-class energy Internet enterprise with global competitiveness, announced that it will fully promote the construction of strong smart grid and ubiquitous power Internet of Things (UPIoT), and transform into a hub, platform and sharer of energy information. To realize the goals, the SGCC should immediately speed up the construction of the UPIoT, a smart service system linking up all things and links in the power system and supporting effective human-machine interaction. This system can be implemented in a conveniently and flexibly to sense all kinds of states and process information in an efficient manner. The functions of the system are realized through various modern information technologies, ranging from the mobile Internet, artificial intelligence to advanced communication technique. In essence, the UPIoT is a wireless sensor network (WSN) established through the application of the IoT technique in power systems [1]. In the WSN, the data are constantly transmitted to the sink node from the sensors. The safety, efficiency and speed of data transmission hinges on the routing optimization [2, 3]. There are many sensors in the UPIoT, some of which are placed under harsh natural conditions. It is difficult to supply power persistently to such sensors. Therefore, it is of great significance to effectively manage the energy consumption of the WSN in the context of the UPIoT.

Planar routing and clustering routing are two kinds of popular routing protocols. The former is too complex, energyintensive and slow in response to be applied in the UPIoT.
Typical examples of planar routing include flooding protocol, sensor protocols for information via negotiation (SPIN) protocol, synthetized adaptive routing (SAR) protocol and directed diffusion mechanism [4]. The clustering routing protocols mainly include the classic low energy adaptive clustering hierarchy (LEACH), the threshold sensitive energy efficiency sensor network protocol (TEEN), and the power efficient gathering in sensor information systems (PEGASIS) $[5,6]$. Among them, the TEEN and PEGASIS are extensions of the LEACH. The clustering routing consumes less energy, responds more quickly and requires easier implementation than the planar routing.

The clustering routing has long been a hot topic among scholars at home and abroad. In Reference [7], a multi-factor clustering algorithm is designed to optimize energy consumption; the algorithm prolongs the network lifecycle through cluster head selection using the fuzzy rule algorithm. In Reference [8], an energy-balanced non-uniform clustering routing algorithm is presented to select cluster heads based on the timer. In Reference [9], an energy-balanced routing algorithm was put forward based on message importance; the algorithm determines the route according to the forwarding income of messages, thus reducing the failure rate and delay of message delivery. Overall, the existing studies fail to consider the limitation of node energy or provide the specific energy consumption model during route optimization or algorithm design, and rarely discusses the WSN route optimization under the UPIoT.

Considering the above, this paper proposes a new rout optimization method for the WSN in the context of the UPIoT. Specifically, the principles of clustering route optimization, 
including cluster head selection and clustering, were set up based on the specific energy consumption model; the clustering principle and the route selection method were combined to ensure the balance of energy consumption among WSN nodes. The experimental results show that the proposed method can effectively reduce the energy consumption of nodes and extend the lifecycle of the entire WSN.

\section{UPIOT}

The UPIoT is an interconnection model for the human, machine and devices inside and outside the grid, which is constructed by applying the 5th generation mobile networks (5G) and the IoT in the power industry. As mentioned before, the UPIoT is also a smart service system that connects all things and all links in the power system, through the integration of modern information technologies like the mobile Internet and artificial intelligence, and advanced communication technique. The system can sense various states and process information efficiently and support effective human-machine interaction, without sacrificing the ease and flexibility in implementation.

The UPIoT typically has a four-layer architecture, namely, a sensing layer, a network layer, a platform layer, and an application layer. The sensing layer relies on widely distributed sensors to collect data from all phases of the grid, including generation, transmission, distribution to consumption, and carries out preliminary data fusion and calculation. Based on $5 \mathrm{G}$ and other modern communication techniques, the network layer connects data and information by setting up a WSN. The platform layer schedules the facilities and processes the big data in the network, preparing for the operations on the application layer. With the goal of safe and stable grid operation, the application layer seeks to build a conservation-oriented, smart and integrated power network.

\section{ENERGY EFFICIENCY MANAGEMENT}

\subsection{Overview}

The UPIoT contains numerous sensor nodes that communicate wirelessly and transmit data via self-organized multi-hop routing $[10,11]$. Due to their small size, the sensors generally have an upper limit on their energy storage. Therefore, it is of critical importance to efficiently use the limited energy in a network consists of numerous sensors, that is, manage the energy efficiency of network nodes. The crux of energy efficiency management is to improve the efficiency of energy use through node management. From the perspective of data transmission, the overall energy loss of the UPIoT can be effectively suppressed by selecting the optimal route from the original nodes to the target node [12]. From the angle of power control of each sensor node, the energy consumption of each node can be reduced if its transmitting and receiving powers are determined based on the size of the local cluster and the distance to its adjacent nodes [13].

Most of the existing methods for energy efficiency management uses an algorithm to minimize the node energy loss without affecting data transmission, and ultimately extend the lifecycle of the entire network [14]. However, the algorithm often makes the data transmission concentrated on a single route, causing energy depletion and premature death to the nodes on that route [15]. To solve the problem, energy balance must be considered in energy efficiency management. In other words, the energy consumption of all nodes should be roughly the same, so that the network will not paralyze due to premature death of a single node.

\subsection{Energy efficiency management model}

The sensor nodes in UPIoT are distributed randomly according to the specific requirements. For each general sensor node, the energy is consumed on three occasions: data transmission, data reception and clustering communication. The data transmission can be further divided into signal emission and signal amplification. The energy consumption of the node is illustrated by the model in Figure 1 below.

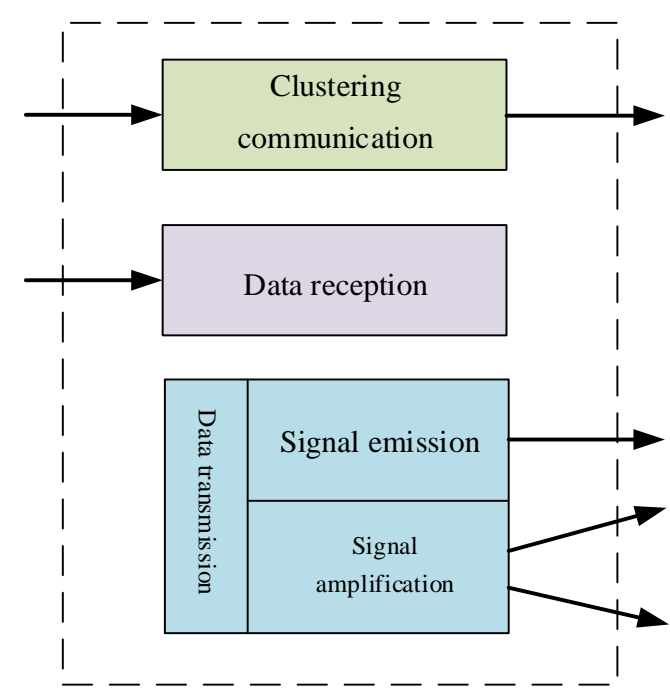

Figure 1. Energy consumption model of each node

During data transmission, the signal emission consumes a constant amount of energy, while the energy consumed to amplify the signal depends on the distance $d$ between two nodes. If the internodal distance $d$ is smaller than the threshold $d_{0}$, the energy consumption is proportional to the square of the distance; if the internodal distance $d$ is greater than the threshold $d_{0}$, the energy consumption is proportional to the fourth power of the distance. The energy consumption of a node to transmit each bit of data can be calculated as:

$$
E_{\text {con }}(d)= \begin{cases}E_{1}+\alpha d^{2}, & d \leq d_{0} \\ E_{1}+\beta d^{4}, & d>d_{0}\end{cases}
$$

where $E_{c o n}(d)$ is the energy consumed to transmit each bit of data; $E_{1}$ is the constant energy consumption of signal emission; $\alpha$ and $\beta$ are the coefficients for the energy consumption of signal amplification under different internodal distances; $d_{0}$ is the threshold of the internodal distance. The threshold can be derived from $\alpha$ and $\beta$ :

$$
d_{0}=\sqrt{\frac{\alpha}{\beta}}
$$

During clustering, the energy consumed by a node is mainly used to communicate with other nodes, that is, the energy consumption depends on the internodal communication. The energy consumed by a node to communicate with $k$ nodes 
with a distance of $d^{*}$ can be expressed as:

$$
E_{c o m}(k, d)=k \eta d^{*}
$$

where $E_{\text {com }}$ is the energy consumed by the node during clustering; $\eta$ is the distance factor of the clustering communication.

Let $E_{\text {rec }}$ be the energy consumed by a node to receive a bit of data. If a node receives $m$ bits of data and transmits $n$ bits of data in a clustering cycle, then the total energy consumption of the node can be computed by:

$$
E_{\text {sum }}=m E_{\text {rec }}+n E_{\text {con }}+E_{\text {com }}
$$

According to the above energy efficiency management model, a more efficient route optimization algorithm can be designed to ensure energy balance of the entire sensor network, thereby maximizing the lifecycle of the sensor network and enhancing its reliability.

\section{WSN ROUTE OPTIMIZATION UNDER THE UPIOT}

\subsection{Overview}

The WSN route optimization mainly tackles the data transmission on the network layer, including route selection and data forwarding $[16,17]$. Route selection refers to finding multiple transmission routes and selecting the optimal route among them, and data forwarding stands for transmitting data long the selected route [18]. Traditionally, the wireless route optimization mostly improves the point-to-point indices like transmission rate and transmission volume. These indices are selected to maximize the traffic and network utilization. In the WSN, however, the multiple nodes each has a limited amount of energy. Hence, the route optimization should focus on the efficient use of the energy in the entire network.

In this paper, the route optimization is carried out based on the clustering routing protocol, one of the two major categories of WSN routing protocols $[19,20]$. The optimization attempts to achieve efficient energy utilization, data fusion, scalability and robustness. The efficient energy utilization is the basic goal of WSN route optimization. On the one hand, the route that consumes the least energy should be selected; on the other hand, the design of the routing algorithm should consider the energy balance among network nodes, avoiding the premature death of any node. The data fusion intends to eliminate data duplication, which is inevitable due to the large number of WSN sensors. The scalability must be considered in route optimization, because some nodes may fail due to the limited node energy and need to be replenished. The WSN must be sufficiently robust to withstand uncertainties arising from the unattendance and harsh environment of many sensor nodes.

\subsection{Cluster head selection based on WSN energy balance}

The cluster head selection is the first step to cluster formation and the precondition for the design of a clustering routing algorithm. In a network, the nodes have basically the same amount of energy at the start, but the cluster heads consume more energy than general nodes. To balance the energy consumption among WSN nodes, the high-quality nodes should be elected as cluster heads. In the WSN, the node quality mainly depends on four factors: the residual energy of the node, the motion velocity of the node, the distance between the node and the sink node, and the distance between the node and its adjacent nodes. The correlations between node quality and the four factors can be described by weight coefficients:

$$
Q=s_{1} \cdot E+s_{2} \cdot V+s_{3} \cdot R_{1}+s_{4} \cdot R_{2}
$$

where $Q$ is the quantified value of node quality; $E$ is the residual energy of the node; $V$ is the motion velocity of the node; $R_{1}$ is the distance between the node and the sink node; $R_{2}$ is the distance between the node and its adjacent nodes; $\mathrm{s}_{1}$, $\mathrm{s}_{2}, \mathrm{~s}_{3}$ and $\mathrm{s}_{4}$ are the weight coefficients of the four factors, respectively. The greater each weight coefficient, the closer the node quality is to the corresponding factor. The four weight coefficients must satisfy the following relationship:

$$
s_{1}+s_{2}+s_{3}+s_{4}=1, s_{1}>s_{2}>s_{3}>s_{4}
$$

Equation (6) shows that the sum of the weight coefficients of the four factors is 1 . By the correlation with node quality, the four factors can be ranked in descending order as the residual energy of the node, the motion velocity of the node, the distance between the node and the sink node, and the distance between the node and its adjacent nodes.

In each cluster, the cluster head is responsible to acquire, screen and fuse the data collected by all the general nodes in the cluster, and send the fused data to the sink node. The reception and fusion process is extremely energy-consuming. As a result, the cluster head consumes more energy than a regular node in the same period of time. For the WSN, if the cluster heads remain unchanged once selected, the network nodes will differ greatly in the residual energy, leading to premature death of cluster heads. In this case, the lifecycle of the entire network will be affected. To prevent the situation, the cluster heads must be replaced timely according to the realtime condition of the nodes. During the replacement, the first two of the above four factors should be considered, i.e. the residual energy of the node $E$ and the motion velocity of the node $V$.

The residual energy of the node $E$ is related to the mean residual energy of all nodes in the cluster:

$$
E=\frac{E_{\text {res }}}{E_{\text {avg }}}
$$

where $E_{\text {res }}$ is the residual energy of the current node; $E_{\text {avg }}$ is the mean residual energy of all nodes in the cluster. The greater the residual energy $E$ of a node, the more likely it is for the node to be selected as the cluster head. If the $E$ value of a cluster head is smaller than one, the cluster head should be replaced by a new node.

The motion velocity of the node $V$ is negatively correlated with the true velocity of the node:

$$
V=\frac{1}{V_{\text {true }}}
$$

where $V_{\text {true }}$ is the true velocity of the node at the current moment. In the WSN, the sensor nodes are generally moving. If moving at a fast velocity, a cluster head will consume lots of energy and may enter other clusters. In this case, more energy will be consumed to transmit the same amount of data from the nodes in the current cluster to the cluster head, and the data may even get lost. Therefore, the motion velocity of the node is an important factor. The greater the true motion 
velocity $V_{\text {true }}$, the smaller the motion velocity $V$, and the less likely for the node to be selected as the cluster head.

The distance between the node to the sink node $R_{1}$ can be calculated by:

$$
R_{1}=1-\frac{l_{i}}{l_{\max }}
$$

where $l_{i}$ is the distance between node $i$ and the sink node; $l_{\max }$ is the maximum distance between the sink node and the nodes within its coverage. In the WSN, the energy consumed to transmit the same amount of data decreases with the distance $l_{i}$ between node $i$ and the sink node. As shown in formula (9), the smaller the $l_{i}$, the greater the $R_{1}$, and the more likely for the node to be selected as the cluster head. Thus, the node with the minimal distance to the sink node should be taken as the cluster head.

The distance between the node and its adjacent nodes $R_{2}$ can be calculated as:

$$
R_{2}=\left(\frac{h_{\min }}{\left.h_{\max }\right)^{2}}\right.
$$

where $h_{\min }$ and $h_{\max }$ are the minimum and maximum distances between the node and its adjacent nodes, respectively. In the WSN, the data transmission from any node in a cluster consumes roughly the same amount of energy if the cluster head is located at similar distances from all general nodes. It can be seen from formula (10) that the value of $R_{2}$ is negatively correlated with the difference between $h_{\min }$ and $h_{\text {max }}$. Therefore, the node with similar distances to all general nodes in the cluster should be taken as the cluster head.

\subsection{WSN node clustering under the UPIoT}

In the WSN, the node clustering is fundamental to the clustering routing protocol. After node clustering, the WSN nodes only need to transmit data to the cluster heads within the specified period, and can turn to the sleep mode and save energy in other periods.

Before node clustering, the cluster heads must be determined by formula (5). Then, each cluster head should broadcast its information to assemble the nearby nodes into its cluster. Note that the sink node, as a special cluster head, should also broadcast its information, and assemble its nearby nodes into a special cluster. In this cluster, the nodes can transmit their data directly to the sink without passing through a cluster head, thus reducing the overall energy loss.

The node density in each cluster should be checked after all the nodes in the WSN have been allocated to different clusters, to avoid excessively high intra-cluster node density. This is because too many nodes in a cluster will push up the energy consumption of the cluster head and the entire cluster, tilting the balance of energy consumption across the network. If a cluster is found to be saturated with nodes, the communication radius of the cluster head can be adjusted to reduce the intracluster node density:

$$
R_{l}=\frac{N_{\text {all }}-N_{\text {part }}}{N_{\text {all }}} \cdot R_{b}
$$

where $R_{l}$ and $R_{b}$ are the adjusted and original communication radii of the cluster head, respectively; $N_{\text {part }}$ is the number of nodes in the cluster to be adjusted; $N_{\text {all }}$ is the total number of nodes to be clustered. The ratio of $N_{\text {part }}$ to $N_{\text {all }}$, i.e. $\frac{N_{\text {part }}}{N_{\text {all }}}$, describes the node density of the cluster to be adjusted.

After its communication radius is adjusted and the communication is stabilized, the cluster head will allocate the time slots for data transmission to the intra-cluster nodes. Then, these nodes only need to transmit data within the allocated slots. In any other time, the nodes should turn off their signal emission and amplification devices to reduce energy consumption. After a period of data transmission, all nodes will update their information like residual energy and motion velocity, and reselect the cluster heads according to formula (5). The node clustering procedure is displayed in Figure 2 below.

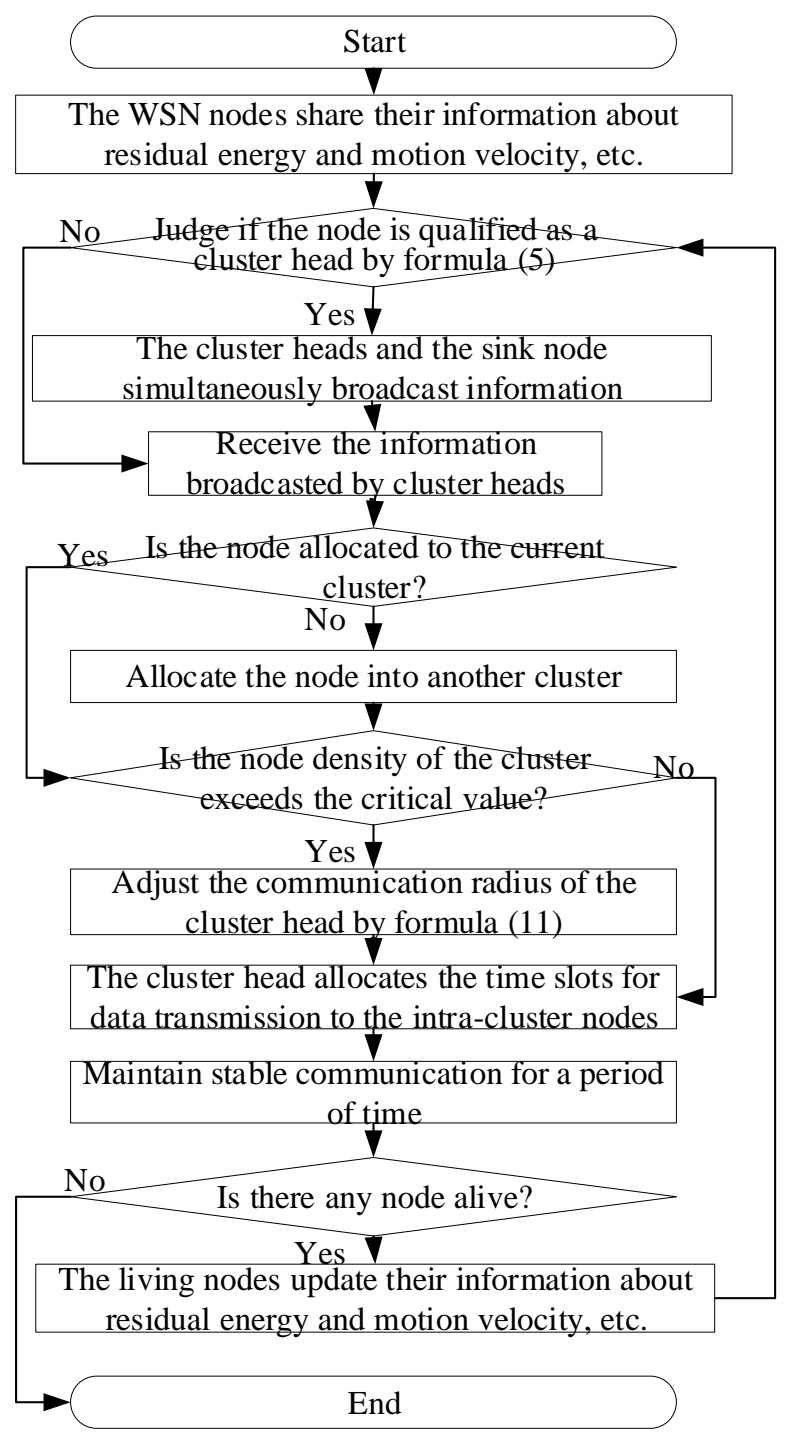

Figure 2. The node clustering procedure

The node clustering of the WSN can be detailed as follows: Step 1: Each node shares with other nodes in the WSN its information about the residual energy, motion velocity, distance to the sink node and distance to the adjacent nodes.

Step 2: Substitute the information shared by a node into formula (5), and judge if the node is a cluster head through comparison.

Step 3: Each node elected as the cluster head broadcasts its identity across the WSN and invites other nodes to join the cluster. The sink node, as a special cluster head, also 
broadcasts its information at the same time. Other regular nodes receive the information broadcasted by cluster heads.

Step 4: Each general node decides whether to enter a cluster based on the distance of the received information. In generally, the general node prefers to join the cluster of a nearby cluster head.

Step 5: After preliminary clustering, judge if the node density of each cluster exceeds the pre-set threshold. If yes, adjust the communication radius of the cluster head by formula (11); otherwise, the cluster head will allocate the time slots for data transmission to the intra-cluster nodes.

Step 6: After a period of stable transmission, judge if there are any node alive in the WSN. If yes, the living nodes will update their information and go to Step 2 for re-clustering; otherwise, terminate the clustering process.

\section{EXPERIMENT DESIGN AND RESULTS ANALYSIS}

\subsection{Experimental preparations}

The proposed routing algorithm was compared with the traditional LEACH routing algorithm through a simulation program designed on the Matlab. In our experiment, the UPIoT-based WSN covers a circular area with a radius of $300 \mathrm{~m}$. In this area, 600 sensor nodes were randomly deployed, and the sink node was placed on the edge of the area.

Before the experiment, all the nodes in the WSN were given an initial energy of $2 \mathrm{~J}$ and a random motion velocity (upper limit: $1 \mathrm{~m} / \mathrm{s}$ ). The data packet length to be transmitted wirelessly between the network nodes was set to $3 \mathrm{kbit}$, the energy consumed by each cluster head to fuse 1 bit of data was set to $0.002 \mathrm{~J}$, and the unadjusted communication radius $R_{l}$ was set to $8 \mathrm{~m}$.

\subsection{Results analysis}

Figure 3 shows the time variation in the number of living nodes in the WSN. The original number of living nodes was 600 .

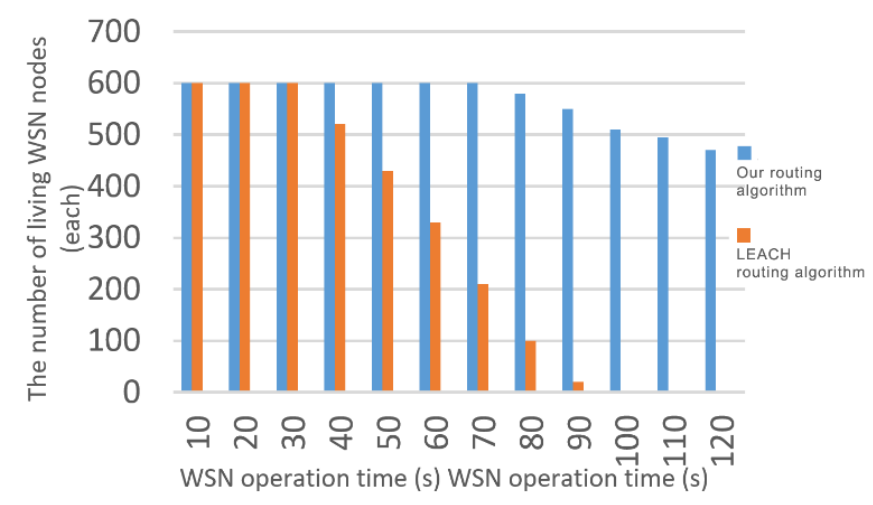

Figure 3. The time variation in the number of living nodes in the WSN

It can be seen that all the nodes in the WSN were alive after the two routing algorithms operated 30s. For the traditional LEACH protocol, the nodes started to die in masses 40s into the operation. The trend continued until all nodes were dead. By contrast, all nodes were alive when our algorithm ran 70s, and only a few deaths were observed since 80 s. This means our routing algorithm can effectively control the node energy and ensure the energy balance among WSN nodes, thus extending the lifecycle of the entire WSN. That is why the first death appeared very late in the experiment.

Figure 4 describes the time variation in the residual energy of single WSN node. The overall trend is that the residual energy decreases with the elapse of time.

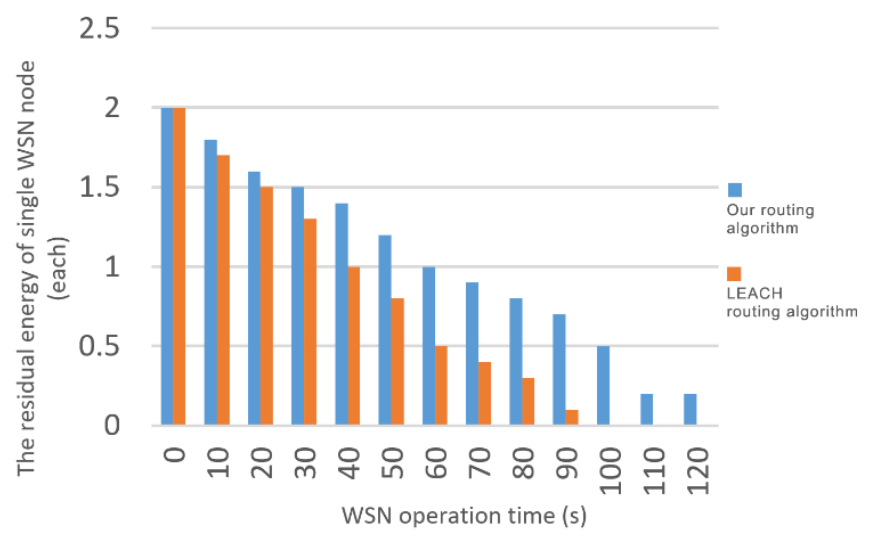

Figure 4. The time variation in the residual energy of single WSN node

As shown in Figure 4, under both routing algorithms, the residual energy of each node gradually decreased with the elapse of time. However, the decline was faster under the traditional LEACH routing algorithm than under our algorithm. In addition, the residual energy dropped to zero, a sign of node death, after 100s of the LEACH operation, while the residual energy was $0.5 \mathrm{~J}$ at the same moment under our algorithm. The above analysis shows that our algorithm can effectively utilize the residual energy and prolong the service life of each node.

\section{CONCLUSIONS}

The UPIoT sets up connections between men and things, as well as between players (e.g. power user, grid enterprise, power enterprise and supplier) and their equipment, and generates a massive amount of shared data to serve the user, the grid, the power enterprise, the supplier, the government and the entire society. Taking the grid as the hub, the UPIoT also acts as a platform and sharer of power information, providing immense opportunities and value services to the entire industry and various market entities. The WSN, the basis of the UPIoT, contains many sensors, some of which are placed under harsh natural conditions. The sensor energy must be fully utilized to improve the data transmission in the WSN under the UPIoT. For this purpose, this paper constructs an energy consumption model for network nodes, and proposes a clustering routing method to extend the lifecycle of the WSN. The Matlab simulation shows our method outperformed the traditional clustering routing algorithm in controlling energy loss of nodes and enhancing network reliability and stability.

\section{REFERENCES}

[1] Liu, C. (2015). Research on WSN cluster head election strategy and routing algorithm based on the leach protocol. Hangzhou Dianzi Universit.

[2] Chen, Y., Xu, X.G., Wang, Y. (2019). Wireless sensor 
network energy efficient coverage method based on intelligent optimization algorithm. Discrete and Continuous Dynamical Systems-Series, 12(4-5): 887900.

[3] Xiao, K.J., Wang, R., Deng, H., Zhang, L., Yang, C.H. (2018). Energy-aware scheduling for information fusion in wireless sensor network surveillance. Information Fusion, 48: 95-106 http://dx.doi.org/10.1016/j.inffus.2018.08.005

[4] Habib, M. (2019). Energy-efficient algorithm for reliable routing of wireless sensor networks. IEEE Transactions on Industrial Electronics, 66(7): 5567-5575.

[5] Caria, M., Jukan, A., Hoffmann, M. (2016). SDN partitioning: A centralized control plane for distributed routing protocols. IEEE Transactions on Network and Service Management, 13(3): 381-393. http://dx.doi.org/10.1109/TNSM.2016.2585759

[6] Al-Shalabi, M., Anbar, M., Wan, T.C. (2018). Variants of the Low-energy adaptive clustering hierarchy protocol: Survey, Issues and Challenges. Electronics, 7(8): 136. http://dx.doi.org/10.3390/electronics7080136

[7] Lee, J.S., Kao, T.Y. (2016). An improved three-layer low-energy adaptive clustering hierarchy for wireless sensor networks. IEEE Internet of Things Journal, 3(6): 951-958. https://doi.org/10.1109/JIOT.2016.2530682

[8] Liu, X.T., Chen, Z.P., Huang, Y.R. (2019). A nonuniform clustering routing algorithm based on energy equilibrium. Microelectronics and Computer, 36(2): 3640.

[9] Chen, Z.G., Yin, B.A., Wu, J. (2018). Message importance based energy balanced routing algorithm. Journal of Communication, 39(12): 91-101.

[10] Asha, G., Santhosh, R. (2019). Soft computing and trustbased self-organized hierarchical energy balance routing protocol (TSHEB) in wireless sensor networks. Soft Computing, 23(8): 2537-2543.

[11] Chowdhury, S., Giri, C. (2019). Energy and network balanced distributed clustering in wireless sensor network. Wireless Personal Communications, 105(3): 1083-1109. https://doi.org/10.1007/s11277-019-06137-Z
[12] Liu, Y.H., Wu, Y.M., Chang, J.Y. (2019). The diffusion clustering scheme and hybrid energy balanced routing protocol (DCRP) in multi-hop wireless sensor networks. AD HOC \& Sensor Wireless Networks, 43(1-2): 33-56.

[13] Kulshrestha, J., Mishra, M.K. (2018). Energy balanced data gathering approaches in wireless sensor networks using mixed-hop communication. Computing, 100(10): 1033-1058. https://doi.org/10.1007/s00607-018-0597-6

[14] Khan, I., Singh, D. (2018). Energy-balance nodeselection algorithm for heterogeneous wireless sensor networks. ETRI Journal, 40(5): 604-612. https://doi.org/10.4218/etrij.2017-0349

[15] Sun, Z.W., Wei, M., Zhang, Z.W. (2019). Secure routing protocol based on multi-objective ant-colonyoptimization for wireless sensor networks. Applied Soft Computing, 77: 366-375.

[16] Mittal, N. (2019). Moth flame optimization based energy efficient stable clustered routing approach for wireless sensor networks. Wireless Personal Communications, 104(2): 677-694. https://doi.org/10.1007/s11277-0186043-4

[17] Tabibi, S., Ghaffari, A. (2019). Energy-efficient routing mechanism for mobile sink in wireless sensor networks using particle swarm optimization algorithm. Wireless Personal Communications, 104(1): 199-216. https://doi.org/10.1007/s11277-018-6015-8

[18] Awad, F.H. (2018). Optimization of relay node deployment for multisource multipath routing in wireless multimedia sensor networks using gaussian distribution. Computer Networks, 145: 96-106.

[19] Ghazi, A., Ahiod, B. (2018). Energy efficient teachinglearning-based optimization for the discrete routing problem in wireless sensor networks. Applied Intelligence, $\quad 48(9)$ : 2755-2769. https://doi.org/10.1007/s10489-017-1108-8

[20] Jayanthi, N., Valluvan, K.R. (2018). Bio-inspired optimization routing technique using DNA sequencing algorithm for wireless sensor networks. Wireless Personal Communications, 101(4): 2365-2381. https://doi.org/10.1007/s11277-018-5821-3 